\begin{tabular}{|c|l|}
\hline Title & A lower bound for fundamental solutions of the heat convection equations \\
\hline Author(s) & Maekawa, Y asunori \\
\hline Citation & Hokkaido University Preprint Series in Mathematics, 788, 1-13 \\
\hline Issue Date & 2006 \\
\hline DOI & 10.14943/83938 \\
\hline Doc URL & http://hdl.handle.net/2115/69596 \\
\hline Type & bulletin (article) \\
\hline File Information & pre788.pdf \\
\hline
\end{tabular}

Instructions for use 


\title{
A LOWER BOUND FOR FUNDAMENTAL SOLUTIONS OF THE HEAT CONVECTION EQUATIONS
}

\author{
YASUNORI MAEKAWA ${ }^{1}$
}

\section{INTRODUCTION}

In this paper we shall consider the diffusion convection equation

$$
\partial_{t} \omega-\Delta \omega+(u, \nabla) \omega=0, t>0, x \in \mathbb{R}^{n} .
$$

Here, $\omega=\omega(t, x)$ is an unknown scalar valued function and $u(t, x)=$ $\left(u^{1}(t, x), \cdots, u^{n}(t, x)\right)$ is a given continuous function satisfying

$$
\left\{\begin{array}{l}
\nabla \cdot u=0, t>0, \text { in the sense of distributions, } \\
\sup _{t>0} t^{\frac{1}{2}}\|u(t, \cdot)\|_{\infty}=M<\infty,
\end{array}\right.
$$

where $\|u(t, \cdot)\|_{\infty}:=\sup _{x \in \mathbb{R}^{n}}|u(t, x)|$. As usual, we set $\partial_{t} \omega=\frac{\partial \omega}{\partial t}, \Delta \omega=$ $\sum_{i=1}^{n} \frac{\partial^{2} \omega}{\partial x_{i}^{2}},(u, \nabla) \omega=\sum_{i=1}^{n} u^{i} \frac{\partial \omega}{\partial x_{i}}$, and $\nabla \cdot u=\sum_{i=1}^{n} \frac{\partial u^{i}}{\partial x_{i}}$.

The equation $\left(A_{u}\right)$ has many applications to problems arising from fluid mechanics. Especially, it can be regarded as the linearized equation of the two dimensional vorticity equation for the incompressible viscous fluid. In this case, $u$ is the velocity field of the fluid. The condition on the norm of $u$ in $(\mathrm{C})$ reflects the rescaling invariant property of the equation. Indeed, let us consider the following rescaling

$$
\begin{aligned}
& \omega_{k}(t, x):=k^{n} \omega\left(k^{2} t, k x\right), \\
& u_{k}(t, x):=k u\left(k^{2} t, k x\right) .
\end{aligned}
$$

If $\omega$ satisfies the equation $\left(A_{u}\right)$, then $\omega_{k}$ satisfies $\left(A_{u_{k}}\right)$. Remark that $u_{k}$ satisfies the condition (C) with the same constant $M$. Such rescaling invariant property is important and often appears when one considers the time global solutions of the nonlinear equations. However, this condition sometimes causes difficulties for the uniqueness and long time behaviors of the solutions, since it gives the critical case whether the convection term $(u, \nabla) \omega$ can be regarded as the perturbation term or not at time zero or at time infinity. To overcome these difficulties, we shall consider the fundamental solutions of $\left(A_{u}\right)$ which are defined as follows.

Definition 1.1. Let $s \geq 0, f \in L^{1}\left(\mathbb{R}^{n}\right)$. Let $u$ satisfy the condition $(C)$. We say that the function $\omega \in C\left((s, \infty) ; L^{1}\left(\mathbb{R}^{n}\right)\right) \cap L^{\infty}\left((s, \infty) ; L^{1}\left(\mathbb{R}^{n}\right)\right)$ is a mild solution of the Cauchy problem:

\footnotetext{
${ }^{1}$ Department of Mathematics, Hokkaido University, Sapporo, 060-0810, Japan. E-mail: yasunori@math.sci.hokudai.ac.jp
} 
$\left(E_{s}\right)$

$$
\left\{\begin{array}{l}
\partial_{t} \omega-\Delta \omega+(u, \nabla) \omega=0, t>s, x \in \mathbb{R}^{n}, \\
\omega(s, x)=f(x), x \in \mathbb{R}^{n}
\end{array}\right.
$$

if and only if $\omega$ satisfies the follwing integral equation

$$
\omega(t)=e^{(t-s) \Delta} f-\int_{s}^{t} \nabla \cdot e^{(t-\tau) \Delta} u(\tau) \omega(\tau) d \tau, t>s .
$$

Here, $e^{t \Delta}$ is the heat semigroup represented as

$$
e^{t \Delta} f=\int_{\mathbb{R}^{n}} G_{t}(x-y) f(y) d y
$$

where

$$
G_{t}(x)=\frac{1}{(4 \pi t)^{\frac{n}{2}}} \exp \left(-\frac{|x|^{2}}{4 t}\right)
$$

Remark 1.1. We do not assume the continuity of mild solutions at initial time in the sense of $L^{1}\left(\mathbb{R}^{n}\right)$. Especially, our results in this paper can be easily extended to the case of finite measures as initial data.

Definition 1.2. Let $s_{0} \geq 0$ and $H_{s_{0}}:=\left\{(t, s, x, y) ; x, y \in \mathbb{R}^{n}, t>s \geq\right.$ $\left.s_{0}\right\}$. We say that the function $\Gamma_{u}(t, x ; s, y)$ is a fundamental solution of $\left(A_{u}\right)$ on $H_{s_{0}}$ if and only if $\Gamma_{u}$ is a function defined on $H_{s_{0}}$ and satisfies that

$$
\begin{aligned}
& \text { (i) } \int_{\mathbb{R}^{n}}\left|\Gamma_{u}(t, x ; s, y)\right| d x, \int_{\mathbb{R}^{n}}\left|\Gamma_{u}(t, x ; s, y)\right| d y<\infty . \\
& (i i) \omega(t, x):=\int_{\mathbb{R}^{n}} \Gamma_{u}(t, x ; s, y) f(y) d y \text { is a mild solution of }\left(E_{s}\right) .
\end{aligned}
$$

Remark 1.2. If $u \equiv 0$, then obviously

$$
\Gamma_{u}(t, x ; s, y)=G_{t-s}(x-y)
$$

is a fundamental solution of $\left(A_{0}\right)$ on $H_{0}$.

The pointwise estimates of fundamental solutions are very useful to study the behaviors of solutions. We already have the upper bound for the fundamental solutions of $\left(A_{u}\right)$ on $H_{s_{0}}$ such as

$$
\Gamma_{u}(t, x ; s, y) \leq \frac{C_{1}}{(t-s)^{\frac{n}{2}}} \exp \left(-C_{2} \frac{|x-y|^{2}}{t-s}\right), t>s \geq s_{0}>0, x, y \in \mathbb{R}^{n},
$$

where $C_{1}, C_{2}$ are positive constants depending only on $n, M$. This estimate was established by E. A. Carlen and M. Loss [3]. S. Matsui and S. Tokuno [10] also obtained the above estimate when $n=2$. Remark that these constants does not depend on $s_{0}>0$. In this paper, we 
shall establish the lower bound for fundamental soutions of $H_{s_{0}}$ by the Gaussian-like function. Our main result is as follows.

Theorem 1.1. Let $s_{0}>0$. Assume that $u$ satisfies (C). Let $\Gamma_{u}$ be the fundamental solution of $\left(A_{u}\right)$ on $H_{s_{0}}$. Then, there exist positive constants $C_{3}, C_{4}$ depending only on $n$ and $M$ (not depending on $s_{0}>0$ ) such that for all $x, y \in \mathbb{R}^{2}, t>s \geq s_{0}$,

$$
\Gamma_{u}(t, x ; s, y) \geq \frac{C_{3}}{(t-s)^{\frac{n}{2}}} \exp \left(-C_{4} \frac{|x-y|^{2}}{t-s}\right)
$$

holds.

The upper and lower pointwise estimates for fundamental solutions by the Gaussian-like functions are called the Aronson estimates. Since the above constants $C_{i}, 1 \leq i \leq 4$ are independent of time variables, these estimates are called the global Aronson estimates. The (global) Aronson estimates were firstly obtained by D. G. Aronson [1] (see also [2]) for the second order parabolic equations of the divergence form:

$$
\partial_{t} \omega-\sum_{1 \leq i, j \leq n} \partial_{i} a_{i j}(t, x) \partial_{j} \omega=0
$$

E. B. Fabes and D. W. Stroock [4] also obtained the global Aronson estimates for fundamental solutions of (5) with different approach using the idea of J. Nash [11]. H. Osada [12] delt with more general type of the divergence form:

$$
\partial_{t} \omega-\sum_{1 \leq i, j \leq n} \partial_{i} a_{i j}(t, x) \partial_{j} \omega+\sum_{i=1}^{n} b_{i}(t, x) \partial_{i} \omega=0,
$$

where $b(t, x)=\left(b_{1}(t, x), \cdots, b_{n}(t, x)\right)$ satisfies

$$
\left\{\begin{array}{l}
\nabla \cdot b=0, t>0, \text { in the sense of distributions, } \\
b_{i}(t, x)=\sum_{j=1}^{n} \partial_{j} c_{i j}(t, x), \text { for some } c_{i j} \in L^{\infty}\left((0, \infty) \times \mathbb{R}^{n}\right) .
\end{array}\right.
$$

In [12], H. Osada showed the existence, the Hölder continuity, and the Aronson estimates of fundamental solutions of (6) with the same methods as in [1] and [2]. These results play important roles in the study of the two dimensional vorticity equations (see [9], [5], and [6]).

As a corollary of our main result, we can prove the well-posedness of mild solutions of $\left(E_{0}\right)$, which seems to be new under the condition $(C)$ on $u$. To see this, note that, as proved in [4] and [11], we can derive the Hölder continuity of fundamental solutions from the upper and lower bounds such as (3) and (4).

Corollary 1.1. Let $s_{0}, d>0$. Assume that $u$ satisfies $(\mathrm{C})$. Let $\Gamma_{u}$ be a fundamental solution of $\left(A_{u}\right)$ on $H_{s_{0}}$. Then, there exists positive constant $C_{5}=C_{5}(n, M, d)$ and $\alpha=\alpha(n, M) \in(0,1)$ such that

$$
\left|\Gamma_{u}(t, x ; s, y)-\Gamma_{u}\left(t^{\prime}, x^{\prime} ; s^{\prime}, y^{\prime}\right)\right| \leq C_{5}\left(\left|x-x^{\prime}\right|^{\alpha}+\left|y-y^{\prime}\right|^{\alpha}+\left|t-t^{\prime}\right|^{\frac{\alpha}{2}}+\left|s-s^{\prime}\right|^{\frac{\alpha}{2}}\right) \text {, }
$$


for all $x, x^{\prime}, y, y^{\prime} \in \mathbb{R}^{n}$ and $t>s \geq s_{0}, t^{\prime}>s^{\prime} \geq s_{0}$ with $|t-s|,\left|t^{\prime}-s^{\prime}\right| \geq$ $d>0$. Especially, the constants $C_{5}$ and $\alpha$ do not depend on $s_{0}$.

The proof of this corollary will be omitted in this paper, since it is obtianed by the same argument of [11]; see section 3 .

Under the condition (C) on $u$, the existence and uniqueness of mild solutions of $\left(E_{s}\right)$ are easily obtained by the iteration argument if $s>0$. Similarly, the existence and uniqueness of fundamental solutions also hold if $s_{0}>0$. Indeed, the existence of a fundamental solution follows by solving the integral equation

$\left.\Gamma_{u}(t, x ; s, y)=G_{t-s}(x-y)-\int_{s}^{t} \int_{\mathbb{R}^{n}} \nabla_{x} \cdot G_{t-\tau}(x-\xi) u(\tau, \xi)\right) \Gamma_{u}(\tau, \xi ; s, y) d \xi d \tau$

and the uniqueness follows from the uniqueness of the mild solution of $\left(E_{s}\right)$. But when $s=0$ (or $s_{0}=0$ ), it seems to be hard to obtain the wellposedness of mild solutions of $\left(E_{0}\right)$ (or funmamental solutions of $\left(A_{u}\right)$ on $H_{0}$, respectively) by the perturbation argument except for the case $M$ is sufficiently small. However, combining the well-posedness for $s>0$ with the above regularity results for fundamental solutions, we can show the well-posedness for $s=0$. That is, we shall prove

Theorem 1.2. Assume that $u$ satisfies (C). Then, there exists a unique fundamental solution of $\left(A_{u}\right)$ on $H_{0}$. Moreover, for any initial data $f \in$ $L^{1}\left(\mathbb{R}^{n}\right)$, the mild solution of $\left(E_{0}\right)$ uniquely exists.

Now let us state the outline of the proof for Theorem 1.1. By using the integral equation (8) and the upper estimate (3), we first prove the estimate (4) for $t>s \geq \kappa t$ with the constant $\kappa \in(0,1)$ depending only on $n$ and $M$. Then, from the semiproup property and the mass conservation law $\int_{\mathbb{R}^{n}} \Gamma_{u}(t, x ; s, y) d x=1$ for fundamental solutions, we obtain the estimate (4) for any $t>s \geq s_{0}$; see section 2 . In section 3 , we give the proof of Theorem 1.2 with some comments for Corollary 1.1. The ingredients of the proof of Theorem 1.2 are the well-posedness of $\left(E_{s}\right)$ for $s>0$, the Hölder continuity and the upper bound of fundamental solutions, and the fact that any mild solution of $\left(E_{0}\right)$ converges to initial data in the sense of measure.

Acknowledgments. The author is grateful to Professor Yoshikazu Giga and Professor Shin'ya Matsui for critical and useful advices.

\section{A LOWER BOUND OF FUNDAMENTAL SOLUTIONS}

Let $s_{0}>0$. We consider a fundamental solution of $\left(A_{u}\right)$ on $H_{s_{0}}$. In this case, by solving the integral equation (8), we have the existence and uniqueness of fundamental solutions. Moreover, we see the following 
translation and rescaling properties.

$$
\begin{aligned}
& \Gamma_{u}(t, x ; s, y)=\Gamma_{u \circ \tau_{y}}(t, x-y ; s, 0), \\
& k^{n} \Gamma_{u}\left(k^{2} t, k x ; k^{2} s, k y\right)=\Gamma_{u_{k}}(t, x ; s, y), k>0,
\end{aligned}
$$

where

$$
\begin{aligned}
& u \circ \tau_{y}(t, x):=u(t, x-y), \\
& u_{k}(t, x):=k u\left(k^{2} t, k x\right) .
\end{aligned}
$$

Remark that if $u$ satisfies (C), then $u \circ \tau_{y}$ and $u_{k}$ also satisfy (C) with the same constant $M$. Now let $t>s \geq s_{0}>0$. Taking $k$ as $k^{2}=s$, we have

$$
\Gamma_{u}(t, x ; s, y)=\Gamma_{u}\left(t, x ; k^{2}, y\right)=\frac{1}{k^{n}} \Gamma_{u_{k}}\left(\frac{t}{k^{2}}, \frac{x}{k} ; 1, \frac{y}{k}\right) .
$$

We set $\frac{t}{k^{2}}=1+\theta, \theta>0, x^{\prime}:=\frac{x}{k}, y^{\prime}:=\frac{y}{k}$. Then it is sufficient to show that there exist positive constants $C_{3}, C_{4}$ depending only on $n$ and $M$ such that for all $x^{\prime}, y^{\prime} \in \mathbb{R}^{n}$ and $\theta>0$,

$$
\Gamma_{u_{k}}\left(1+\theta, x^{\prime} ; 1, y^{\prime}\right) \geq \frac{C_{3}}{\theta^{\frac{n}{2}}} e^{-C_{4} \frac{\left|x^{\prime}-y^{\prime}\right|^{2}}{\theta}} .
$$

Since the rescaled function $u_{k}$ satisfies the same condition as $u$, we write $u$ instead of $u_{k}$. The following proposition is essential in this paper.

Proposition 2.1. There exists $\kappa \in(0,1)$ depending only on $n$ and $M$ such that for any $\kappa \theta \leq s<t \leq \theta$ and $x, y \in \mathbb{R}^{n}$ with $|x-y| \leq(t-s)^{\frac{1}{2}}$, the estimate

$$
\Gamma_{u}(1+t, x ; 1+s, y) \geq \frac{C_{0}}{(t-s)^{\frac{n}{2}}}
$$

holds. Here we can take $C_{0}$ as $C_{0}=\frac{1}{2(4 \pi)^{\frac{n}{2}}} e^{-\frac{1}{4}}$.

Proof: We consider the integral equation which $\Gamma_{u}(1+t, x ; 1+s, y)$ satisfies.

$$
\begin{aligned}
& \Gamma_{u}(1+t, x ; 1+s, y) \\
= & G_{t-s}(x-y) \\
& -\int_{1+s}^{1+t} \int_{\mathbb{R}^{n}} \nabla_{x} \cdot G_{1+t-\tau}(x-\xi) u(\tau, \xi) \Gamma_{u}(\tau, \xi ; 1+s, y) d \xi d \tau .
\end{aligned}
$$


We estimate the second term of (14). From the estimate (3), we have

$$
\begin{aligned}
& \left|\int_{1+s}^{1+t} \int_{\mathbb{R}^{n}} \nabla_{x} \cdot G_{1+t-\tau}(x-\xi) u(\tau, \xi) \Gamma_{u}(\tau, \xi ; 1+s, y) d \xi d \tau\right| \\
\leq & \int_{1+s}^{1+t} \int_{\mathbb{R}^{n}} C \frac{|x-\xi|}{(1+t-\tau)^{\frac{n}{2}+1}} e^{-\frac{|x-\xi|^{2}}{4(1+t-\tau)}}|u(\tau, \xi)| \Gamma_{u}(\tau, \xi ; 1+s, y) d \xi d \tau \\
\leq & C C_{1} M \int_{1+s}^{1+t} \frac{1}{(1+t-\tau)^{\frac{n+1}{2}}(\tau-1-s)^{\frac{n}{2}} \tau^{\frac{1}{2}}} \int_{\mathbb{R}^{n}} e^{-\frac{|x-\xi|^{2}}{8(1+t-\tau)}} e^{-C_{2} \frac{|y-\xi|^{2}}{\tau-1-s}} d \xi d \tau \\
\leq & \frac{1}{(t-s)^{\frac{n}{2}}} \int_{1+s}^{1+t} \frac{1}{(1+t-\tau)^{\frac{1}{2}} \tau^{\frac{1}{2}}} d \tau \\
\leq & \frac{\tilde{C}}{(1+s)^{\frac{1}{2}}(t-s)^{\frac{n-1}{2}}},
\end{aligned}
$$

where $\tilde{C}$ is a positive constant depending only on $n$ and $M$.

Hence, if $|x-y| \leq(t-s)^{\frac{1}{2}}$, then we have

$$
\begin{aligned}
& \Gamma_{u}(1+t, x ; 1+s, y) \\
\geq & \frac{e^{-\frac{|x-y|^{2}}{4(t-s)}}}{\{4 \pi(t-s)\}^{\frac{n}{2}}}-\frac{\tilde{C}}{(1+s)^{\frac{1}{2}}(t-s)^{\frac{n-1}{2}}} \\
\geq & \frac{e^{-\frac{1}{4}}}{\{4 \pi(t-s)\}^{\frac{n}{2}}}-\frac{\tilde{C}}{(1+s)^{\frac{1}{2}}(t-s)^{\frac{n-1}{2}} .}
\end{aligned}
$$

Set $K:=4(4 \pi)^{n} \tilde{C}^{2} e^{\frac{1}{2}}$ and $\kappa=\frac{K}{1+K}$. By elementary calculation, if $\kappa \theta \leq s<t \leq \theta$, then we have

$$
\frac{e^{-\frac{1}{4}}}{2\{4 \pi(t-s)\}^{\frac{n}{2}}} \geq \frac{\tilde{C}}{(1+s)^{\frac{1}{2}}(t-s)^{\frac{n-1}{2}}} .
$$

Thus, for $|x-y| \leq(t-s)^{\frac{1}{2}}$, the estimate

$$
\Gamma_{u}(1+t, x ; 1+s, y) \geq \frac{e^{-\frac{1}{4}}}{2\{4 \pi(t-s)\}^{\frac{n}{2}}},
$$

holds, which is the desired estimate.

Remark 2.1. In the above proof, we essentially use the upper bound for fundamental solutions (3), which was obtained by E. A. Carlen and M. Loss [3], and S. Matsui and S. Tokuno [10]. In fact, [10] studies the two dimensional case under the further regularity condition on $u(t, \cdot) \in$ $C^{1}\left(\mathbb{R}^{n}\right)$ for each $t \in(0, \infty)$. But there seems to be a small flaw in the argument in $[10]$. They consider the operator $F_{u}(t, s): L^{2}\left(\mathbb{R}^{2}\right) \rightarrow L^{\infty}\left(\mathbb{R}^{2}\right)$ defined as

$$
F_{u}(t, s) f:=e^{-\alpha \cdot x} \int_{\mathbb{R}^{2}} \Gamma_{u}(t, x ; t-s, y) e^{\alpha \cdot y} d y, \alpha \in \mathbb{R}^{2} .
$$


In their proof, the fundamental solution which defines the adjoint operator $\left(F_{u}(t, s)\right)^{*}: L^{1}\left(\mathbb{R}^{2}\right) \rightarrow L^{2}\left(\mathbb{R}^{2}\right)$ is set as $\Gamma_{-u}(s, y ; 0, x)[10$, p.537]. However, in fact, this fundamental solution must be $\Gamma_{u \circ \tau_{t}}(s, y ; 0, x)$, where $u \circ \tau_{t}(s, x):=u(t-s, x)$. One of the keys in their argument is that $\left(F_{u}(t, s)\right)^{*}$ has the same estimates as those of $F_{u}(t)$. But, one cannot apply the estimate for $F_{u}(t)$ directly to get the estimate for $\left(F_{u}(t, s)\right)^{*}$ since the condition for $v(s, x):=u \circ \tau_{t}(s, x)$ is $\sup _{0<s<t}(t-s)^{\frac{1}{2}}\|v(s, \cdot)\|_{L^{\infty}}<\infty$ instead of $(C)$. Fortunately, by arguing the same way as for $F_{u}(t, s)$, one can check that $\left(F_{u}(t, s)\right)^{*}$ has the same type estimate of the operator norm as $F_{u}(t, s)$ has, so their estimate for $\Gamma_{u}$ is correct.

In [3], E. A. Carlen and M. Loss deal with more general type of the convection term. Especially, they consider the equation of the type

$$
\partial_{t} \omega(t, x)-\Delta \omega(t, x)+\nabla \cdot(f(x, \omega) \omega(t, x))=0,
$$

where $f$ satisfies $\nabla \cdot f(\cdot, \omega(\cdot, t))=0$ and $\|f(\cdot, \omega(\cdot, t))\|_{\infty} \leq B(t)$ for some $B(t) \in L^{1}(0, T), \forall T>0$. They obtained the estimate such as

$$
G(t, x ; 0, y) \leq(4 \pi t)^{-\frac{n}{2}} \exp \left[-\frac{|x-y|^{2}}{4 t}\left(1-\frac{1}{|x-y|} \int_{0}^{t} B(\tau) d \tau\right)_{+}^{2}\right]
$$

where $G(t, x ; 0, y)$ is a fundamental solution of (16) (if it exists), and $(\cdot)_{+}=\max (\cdot, 0) ;$ see $[3$, Theorem 3$]$.

Once we obtain the estimate (13), we can prove the lower Aronson estimate of $\Gamma_{u}(1+t, x ; 1+s, y)$ at least for $\kappa \theta \leq s<t \leq \theta$ by using the argument in [1] (see also [4]).

Proposition 2.2. For any $\kappa \theta \leq s<t \leq \theta$ and $x, y \in \mathbb{R}^{n}$, we have

$$
\Gamma_{u}(1+t, x ; 1+s, y) \geq \frac{C}{(t-s)^{\frac{n}{2}}} e^{-C^{\prime} \frac{|x-y|^{2}}{t-s}}, x, y \in \mathbb{R}^{n}
$$

where $C, C^{\prime}$ are numerical constants (not depending even on $M$ ).

Proof: The following argument for the above proposition is by D. G. Aronson [1]. From (9), we may assume that $y=0$ without loss of generality. Let $|x|>(t-s)^{\frac{1}{2}}$ and let $m$ be the smallest integer dominating $\frac{4|x|^{2}}{t-s}$ and set $S=\prod_{l=1}^{m-1} B\left(\frac{l}{m} x ; \frac{1}{4} \sqrt{\frac{t-s}{m}}\right)$, where $B(y ; r):=\left\{\xi \in \mathbb{R}^{n} ; \mid \xi-\right.$ $y \mid<r\}$. Then we can easily check that for $\left(\xi_{1}, \ldots, \xi_{m-1}\right) \in S$, we have

$$
\left|\xi_{1}\right|<\sqrt{\frac{t-s}{m}}, \max _{1<l<m}\left|\xi_{l}-\xi_{l-1}\right| \leq \sqrt{\frac{t-s}{m}},\left|x-\xi_{m-1}\right|<\sqrt{\frac{t-s}{m}}
$$


Then we have from (13) and the semigroup property of the fundamental solution,

$$
\begin{aligned}
& \Gamma_{u}(1+t, x ; 1+s, 0) \\
= & \int \ldots \int \Gamma_{u}\left(1+t, x ; 1+t-\frac{t-s}{m}, \xi_{1}\right) \\
& \ldots \Gamma_{u}\left(1+t-\frac{l(t-s)}{m}, \xi_{l} ; 1+t-\frac{(l+1)(t-s)}{m}, \xi_{l+1}\right) \ldots \\
& \ldots \Gamma_{u}\left(1+t-\frac{(k-1)(t-s)}{m}, \xi_{m-1} ; 1+s, 0\right) d \xi_{m-1} \ldots d \xi_{1} \\
\geq & \int \ldots \int_{S} \Gamma_{u}\left(1+t, x ; 1+t-\frac{t-s}{m}, \xi_{1}\right) \\
& \ldots \Gamma_{u}\left(1+t-\frac{l(t-s)}{m}, \xi_{l} ; 1+t-\frac{(l+1)(t-s)}{m}, \xi_{l+1}\right) \ldots \\
& \ldots \Gamma_{u}\left(1+t-\frac{(m-1)(t-s)}{m}, \xi_{m-1} ; 1+s, 0\right) d \xi_{m-1} \ldots d \xi_{1} \\
\geq & \left(\frac{C_{0} m}{(t-s)^{\frac{n}{2}}}\right)^{m}|S| \\
= & \left(\frac{C_{0} m}{(t-s)^{\frac{n}{2}}}\right)^{m}\left(\pi \frac{t-s}{16 m}\right)^{m-1} \\
= & \frac{C_{0} m}{(t-s)^{\frac{n}{2}}}\left(\frac{C_{0} \pi}{16}\right)^{m-1} \\
\geq & \frac{C_{0}}{(t-s)^{\frac{n}{2}}} e^{-\eta(m-1)} \geq \frac{C_{0}}{(t-s)^{\frac{n}{2}}} e^{-4 \eta \frac{|x|^{2}}{t-s}},
\end{aligned}
$$

where $1>\frac{C_{0} \pi}{16}=: e^{-\eta}$. So (18) holds.

Finally, we extend the estimate (18) to $s=0$. To see this, note that the mass conservation law

$$
\int_{\mathbb{R}^{n}} \Gamma_{u}(t, x ; s, y) d x=1
$$

holds. This is directly established from the integral equation (8), since we have

$$
\begin{aligned}
& \left.\int_{\mathbb{R}^{n}} \int_{s}^{t} \int_{\mathbb{R}^{n}} \nabla_{x} \cdot G_{t-\tau}(x-\xi) u(\tau, \xi)\right) \Gamma_{u}(\tau, \xi ; s, y) d \xi d \tau d x \\
= & \left.\int_{s}^{t} \int_{\mathbb{R}^{n}} \int_{\mathbb{R}^{n}} \nabla_{x} \cdot G_{t-\tau}(x-\xi) u(\tau, \xi)\right) \Gamma_{u}(\tau, \xi ; s, y) d x d \xi d \tau \\
= & 0,
\end{aligned}
$$

and

$$
\int_{\mathbb{R}^{n}} G_{t-s}(x-y) d x=1
$$


Proof of the estimate (12):

Again we may assume that $y=0$. From the estimate (18), we have

$$
\begin{aligned}
& \Gamma_{u}(1+\theta, x ; 1,0) \\
= & \int_{\mathbb{R}^{n}} \Gamma_{u}(1+\theta, x ; 1+\kappa \theta, \xi) \Gamma_{u}(1+\kappa \theta, \xi ; 1,0) d \xi \\
\geq & \int_{\mathbb{R}^{n}} \frac{C}{\{(1-\kappa) \theta\}^{\frac{n}{2}}} e^{-C^{\prime} \frac{|x-\xi|^{2}}{(1-\kappa) \theta}} \Gamma_{u}(1+\kappa \theta, \xi ; 1,0) d \xi,
\end{aligned}
$$

and since $|x-\xi|^{2} \leq 2\left(|x|^{2}+|\xi|^{2}\right)$, we have

$$
\begin{aligned}
& \geq \frac{C}{\{(1-\kappa) \theta\}^{\frac{n}{2}}} e^{-2 C^{\prime} \frac{|x|^{2}}{(1-\kappa) \theta}} \int_{\mathbb{R}^{n}} e^{-2 C_{2} \frac{|\xi|^{2}}{(1-\kappa) \theta}} \Gamma_{u}(1+\kappa \theta, \xi ; 1,0) d \xi \\
& \geq \frac{C}{\{(1-\kappa) \theta\}^{\frac{n}{2}}} e^{-2 C^{\prime} \frac{|x|^{2}}{(1-\kappa) \theta}} \int_{|\xi| \leq R \sqrt{\theta}} e^{-2 C^{\prime} \frac{|\xi|^{2}}{(1-\kappa) \theta}} \Gamma_{u}(1+\kappa \theta, \xi ; 1,0) d \xi \\
& \geq \frac{C}{\{(1-\kappa) \theta\}^{\frac{n}{2}}} e^{-\frac{2 C^{\prime} R^{2}}{1-\kappa}} e^{-2 C^{\prime} \frac{|x|^{2}}{(1-\kappa) \theta}} \int_{|\xi| \leq R \sqrt{\theta}} \Gamma_{u}(1+\kappa \theta, \xi ; 1,0) d \xi,
\end{aligned}
$$

where $R$ is an any positive number. So from (19) and the upper estimate (3),

$$
\begin{aligned}
& \Gamma_{u}(1+\theta, x ; 1,0) \\
\geq & \frac{C}{\{(1-\kappa) \theta\}^{\frac{n}{2}}} e^{-\frac{2 C^{\prime} R^{2}}{1-\kappa}} e^{-2 C^{\prime} \frac{|x|^{2}}{(1-\kappa) \theta}}\left(1-\int_{|\xi| \geq R \sqrt{\theta}} \Gamma_{u}(1+\kappa \theta, \xi ; 1,0) d \xi\right) \\
\geq & \frac{C}{\{(1-\kappa) \theta\}^{\frac{n}{2}}} e^{-\frac{2 C^{\prime} R^{2}}{1-\kappa}} e^{-2 C^{\prime} \frac{|x|^{2}}{(1-\kappa) \theta}}\left(1-\int_{|\xi| \geq R \sqrt{\theta}} \frac{C_{1}}{(\kappa \theta)^{\frac{n}{2}}} e^{-C_{2} \frac{|\xi|^{2}}{\kappa \theta}} d \xi\right) \\
= & \frac{C}{\{(1-\kappa) \theta\}^{\frac{n}{2}}} e^{-\frac{2 C^{\prime} R^{2}}{1-\kappa}} e^{-2 C^{\prime} \frac{|x|^{2}}{(1-\kappa) \theta}}\left(1-\int_{|z| \geq R} \frac{C_{1}}{\kappa^{\frac{n}{2}}} e^{-C_{2} \frac{|z|^{2}}{\kappa}} d z\right) \\
\geq & \frac{C}{2\{(1-\kappa) \theta\}^{\frac{n}{2}}} e^{-\frac{2 C^{\prime} R^{2}}{1-\kappa}} e^{-2 C^{\prime} \frac{|x|^{2}}{(1-\kappa) \theta}}
\end{aligned}
$$

if $R$ is sufficiently large. Remark that the choice of $R$ does not depend on $\theta$ and $x$. This proves the estimate (12). Hence, by rescaling, we obtain the estimate (4), which proves the main thorem.

\section{Well-posedness of the Cauchy problem $\left(E_{0}\right)$}

In this section, we consider the existence and uniqueness of mild solutions of $\left(E_{0}\right)$. Before giving the proof for Theorem 1.2, we note that Corollary 1.1 follows by arguing the same way as in J. Nash [11]. Although the equations studied in [11] are of divergence form (5) (so there is no lower order term), the tools used there in order to obtain the Hölder 
continuity of the fundamental solution are just as follows.

(i)semigroup property of the fundamental solution,

(ii) $\int_{\mathbb{R}^{n}} S(t, x ; s, y) d x=\int_{\mathbb{R}^{n}} S(t, x ; s, y) d y=1$

$($ iii $) C(t-s)^{\frac{1}{2}} \leq \int_{\mathbb{R}^{n}}|x-y| S(t, x ; s, y) d x \leq C^{\prime}(t-s)^{\frac{1}{2}}$,

(iv)for some $K>0$,

$$
\left.\int_{\mathbb{R}^{n}} \min \left\{S\left(t, x ; s, y_{1}\right)\right), S\left(t, x ; s, y_{2}\right)\right\} d x \geq \phi\left(\frac{\left|y_{1}-y_{2}\right|}{(t-s)^{\frac{1}{2}}}\right), \text { if } \frac{\left|y_{1}-y_{2}\right|}{(t-s)^{\frac{1}{2}}} \leq K
$$

where $S$ is the fundamental solution of (5), $C, C^{\prime}$ are positive constants independent of $t, s, x, y$, and $\phi$ is a positive and decreasing function. Once we obtain the above properties for the fundamental solution $\Gamma_{u}$, we can apply the argument of [11, p.943-948], from which we obtain the Hölder continuity of $\Gamma_{u}$. It is not difficult to see the properties $(i)-(i i i)$ hold if $t>s \geq s_{0}>0$. The property $(i v)$ is also obtained by the same argument as in [11, p.943-944]. However, we give the proof here for convenience to reader. First, from the lower bound (4), we have

$$
\int_{\mathbb{R}^{n}} G_{t-s}(x-y) \log \left\{(t-s)^{\frac{n}{2}} \Gamma_{u}(t, x ; s, y)\right\} d x \geq-C,
$$

where $C$ is a positive constant depending only on $n, M$. This implies the estimate

$$
\begin{aligned}
& \int_{\mathbb{R}^{n}} \max _{i}\left[G_{t-s}\left(x-y_{i}\right)\right] \max _{i}\left[\log \left\{(t-s)^{\frac{n}{2}} \Gamma_{u}\left(t, x ; s, y_{i}\right)\right\}\right] d x \\
& \quad+\int_{\mathbb{R}^{n}} \min _{i}\left[G_{t-s}\left(x-y_{i}\right)\right] \min _{i}\left[\log \left\{(t-s)^{\frac{n}{2}} \Gamma_{u}\left(t, x ; s, y_{i}\right)\right\}\right] d x \\
& \geq \quad-2 C, y_{i} \in \mathbb{R}^{n}, i=1,2 .
\end{aligned}
$$

Using the inequality $\log x \leq x-1$ and $(i i)$, we have

$$
\begin{aligned}
& \int_{\mathbb{R}^{n}} \max _{i}\left[G_{t-s}\left(x-y_{i}\right)\right] \max _{i}\left[\log \left\{(t-s)^{\frac{n}{2}} \Gamma_{u}\left(t, x ; s, y_{i}\right)\right\}\right] d x \\
\leq & \int_{\mathbb{R}^{n}} \max _{i}\left[G_{t-s}\left(x-y_{i}\right)\right] \max _{i}\left[\left\{(t-s)^{\frac{n}{2}} \Gamma_{u}\left(t, x ; s, y_{i}\right)\right\}\right] d x \\
\leq & \int_{\mathbb{R}^{n}} \Gamma_{u}\left(t, x ; s, y_{1}\right)+\Gamma_{u}\left(t, x ; s, y_{2}\right) d x \\
= & 2
\end{aligned}
$$


and

$$
\begin{aligned}
& \int_{\mathbb{R}^{n}} \min _{i}\left[G_{t-s}\left(x-y_{i}\right)\right] \min _{i}\left[\log \left\{(t-s)^{\frac{n}{2}} \Gamma_{u}\left(t, x ; s, y_{i}\right)\right\}\right] d x \\
\leq & \int_{\mathbb{R}^{n}} \min _{i}\left[G_{t-s}\left(x-y_{i}\right)\right] \min _{i}\left[\log \left\{(t-s)^{\frac{n}{2}}\left(\Gamma_{u}\left(t, x ; s, y_{i}\right)\right\}+\delta\right] d x\right. \\
\leq & w \log \delta+\int_{\mathbb{R}^{n}} \min _{i}\left[G_{t-s}\left(x-y_{i}\right)\right] \min _{i}\left[\log \left(1+\delta^{-1}\left\{(t-s)^{\frac{n}{2}} \Gamma_{u}\left(t, x ; s, y_{i}\right)\right\}\right)\right] d x \\
\leq & w \log \delta+\delta^{-1} \int_{\mathbb{R}^{n}} \min _{i}\left[\Gamma_{u}\left(t, x ; s, y_{i}\right)\right] d x,
\end{aligned}
$$

for any $\delta>0$. Here, $w:=\int_{\mathbb{R}^{n}} \min _{i}\left[G_{t-s}\left(x-y_{i}\right)\right] d x$, which is a function of $\frac{\left|y_{1}-y_{2}\right|}{(t-s)^{\frac{1}{2}}}$. Thus, we have

$$
\begin{aligned}
& \int_{\mathbb{R}^{n}} \min _{i}\left[\Gamma_{u}\left(t, x ; s, y_{i}\right)\right] d x \\
\geq & \delta(-2-2 C-w \log \delta)=: \phi\left(\frac{\left|y_{1}-y_{2}\right|}{(t-s)^{\frac{1}{2}}}\right) .
\end{aligned}
$$

Remark that the function $w$ is positive and bounded away from zero if $\frac{\left|y_{1}-y_{2}\right|}{(t-s)^{\frac{1}{2}}}$ is not large. So the above $\phi$ is positive and decreasing if we choose $\delta$ sufficiently small. This shows $(i v)$ holds and we have Corollary 1.1.

Let $t>s>0$ and let $\Gamma_{u}(t, x ; s, y)$ be a (unique) fundamental solution. For each fixed $t, x, y$, from the Hölder coninuity with respect to $s$, we can uniquely extend $\Gamma_{u}$ up to $s=0$. That is, we define the function $\Gamma_{u}(t, x ; 0, y)$ by

$$
\Gamma_{u}(t, x ; 0, y):=\lim _{s \rightarrow 0} \Gamma_{u}(t, x ; s, y) .
$$

It is easy to see that $\Gamma_{u}(t, x ; 0, y)$ satisfies the integral equation (8) with $s=0$ and this shows that $\Gamma_{u}(t, x ; 0, y)$ is a unique fundamental solution of $\left(A_{u}\right)$ on $H_{0}$. Remark that $\Gamma_{u}(t, x ; 0, y)$ satisfies that

$$
\begin{aligned}
& \Gamma_{u}(t, x ; 0, y)=\int_{\mathbb{R}^{n}} \Gamma_{u}(t, x ; s, \xi) \Gamma_{u}(s, \xi ; 0, y) d \xi, s>0, \\
& \frac{C_{3}}{t^{\frac{n}{2}}} e^{-C_{4} \frac{|x-y|^{2}}{t}} \leq \Gamma_{u}(t, x ; 0, y) \leq \frac{C_{1}}{t^{\frac{n}{2}}} e^{-C_{2} \frac{|x-y|^{2}}{t}} .
\end{aligned}
$$

The existence of the fundamental solution on $H_{0}$ implies the existence of a mild solution of $\left(E_{0}\right)$. Thus it suffices to prove the uniqueness of mild solutions of $\left(E_{0}\right)$. First, we claim the following assertion.

Proposition 3.1. Let $\omega$ be any mild solution of $\left(E_{0}\right)$ with initial data $f \in L^{1}\left(\mathbb{R}^{n}\right)$. Then, $\omega(t, \cdot)$ converges to $f$ as $t$ goes to zero in the sense of measure.

The above proposition easily follows from the integral equation (1). We omit the details. 


\section{Proof of the uniqueness of the mild solution of $\left(E_{0}\right)$.}

Let $\omega$ be any mild solution of $\left(E_{0}\right)$ with intial data $f \in L^{1}\left(\mathbb{R}^{n}\right)$ and let $\tilde{\omega}$ be the function defined as

$$
\tilde{\omega}(t, x):=\int_{\mathbb{R}^{n}} \Gamma_{u}(t, x ; 0, y) f(y) d y .
$$

It suffices to prove that $\omega(t, x)=\tilde{\omega}(t, x)$. Since the uniqueness of the mild solution of $\left(E_{s}\right)$ holds for any $s>0, \omega(t, \cdot)$ can be regarded as the unique mild solution of $\left(E_{s}\right)$ with initial data $\omega(s, \cdot)$ for any $t>s>0$. In other words, $\omega(t, x)$ can be represented as

$$
\omega(t, x)=\int_{\mathbb{R}^{n}} \Gamma_{u}(t, x ; s, y) \omega(s, y) d y .
$$

Fix $t$ and $x$. Let $\varphi_{R}(r)$ be a smooth function on $[0, \infty)$ such that $0 \leq$ $\varphi_{R}(r) \leq 1$ and

$$
\varphi_{R}(r)=\left\{\begin{array}{l}
1, \text { if } r<R, \\
0, \text { if } r>2 R .
\end{array}\right.
$$

We set $a_{R}^{(t, x)}(y):=\varphi_{R}\left(\frac{|x-y|^{2}}{t}\right)$ and $b_{R}^{(t, x)}(y)=1-a_{R}^{(t, x)}(y)$. From the Hölder continuity of the fundamental solution, we have

$$
\begin{aligned}
& |\omega(t, x)-\tilde{\omega}(t, x)| \\
= & \left|\int_{\mathbb{R}^{n}} \Gamma_{u}(t, x ; s, y) \omega(s, y) d y-\int_{\mathbb{R}^{n}} \Gamma_{u}(t, x ; 0, y) f(y) d y\right| \\
\leq & \left|\int_{\mathbb{R}^{n}}\left(\Gamma_{u}(t, x ; s, y)-\Gamma_{u}(t, x ; 0, y)\right) \omega(s, y) d y\right|+\left|\int_{\mathbb{R}^{n}} \Gamma_{u}(t, x ; 0, y)(\omega(s, y)-f(y)) d y\right| \\
\leq & \left.C s^{\frac{\alpha}{2}} \sup _{t>0}|| \omega(t, \cdot)\right|_{L^{1}}+\left|\int_{\mathbb{R}^{n}} a_{R}^{(t, x)}(y) \Gamma_{u}(t, x ; 0, y)(\omega(s, y)-f(y)) d y\right| \\
& +\left|\int_{\mathbb{R}^{n}} b_{R}^{(t, x)}(y) \Gamma_{u}(t, x ; 0, y)(\omega(s, y)-f(y)) d y\right| \\
\leq & C s^{\frac{\alpha}{2}} \sup _{t>0}|| \omega(t, \cdot)||_{L^{1}}+\left|\int_{\mathbb{R}^{n}} a_{R}^{(t, x)}(y) \Gamma_{u}(t, x ; 0, y)(\omega(s, y)-f(y)) d y\right| \\
& +\int_{\frac{|x-y|^{2}}{t} \geq R} b_{R}^{(t, x)}(y) \Gamma_{u}(t, x ; 0, y)(|\omega(s, y)|+|f(y)|) d y .
\end{aligned}
$$

By the upper estimate (3), if $\frac{|x-y|^{2}}{t} \geq R$, then

$$
\Gamma_{u}(t, x ; 0, y) \leq \frac{C_{1}}{t^{\frac{n}{2}}} e^{-C_{2} R} .
$$

Since $\omega(s, \cdot)$ converges to $f$ as $s$ goes to zero in the sense of measure, we have

$$
\begin{aligned}
|\omega(t, x)-\tilde{\omega}(t, x)| & \leq \frac{C_{1}}{t^{\frac{n}{2}}} e^{-C_{2} R}\left(\sup _{t>0}\|\omega(t, \cdot)\|_{L^{1}}+\|f\|_{L^{1}}\right) \\
& \rightarrow 0 \text { as } R \rightarrow \infty .
\end{aligned}
$$


This completes the proof of Theorem 1.2.

Remark 3.1. From the proof above, we see that the uniqueness of the mild solution also follows for finite measures as initial data.

\section{REFERENCES}

[1] Aronson, D. G. Bounds for fundamental solutions of a parabolic equation. Bull. Amer. Math. Soc. 1968, 73, 890-896.

[2] Aronson, D. G.; Serrin, J. Local behavior of solutions of quasilinear parabolic equations. Arch. Rational Mech. Anal. 1967, 25, 81-122.

[3] Carlen, E. A.; Loss, M. Optimal smoothing and decay estimates for viscously damped conservation laws, with applications to the 2-D Navier-Stokes equation. Duke Math. J. 1996, 81, 135-157.

[4] Fabes, E. B.; Strook, D. W. A new proof of Moser's parabolic Harnack inequality using the old idea of Nash. Arch. Rational Mech. Anal. 1986, 96, 327-338.

[5] Gallay, Th.; Wayne, C. E. Global stability of vortex solutions of the twodimentional Navier-Stokes equation. Comm. Math. Phys., 2005, 255, no.1, 97129 .

[6] Gallagher, I.; Gallay, Th. Uniqueness for the two-dimensional Navier-Stokes equation with a measure as initial vorticity., Math. Ann., 2005, 332, no.2, 287327.

[7] Giga, Y.; Kambe, T. Large time behavior of the vorticity of two dimensional viscous flow and its application to vortex formation. Comm. Math. Phys. 1988, $117,549-568$.

[8] Giga, Y.; Giga, M.-H. Nonlinear Partial Differential Equation, Self-similar solutions and asymptotic behavior; Kyoritsu: 1999 (in Japanese), English version to be published by Birkhäuser

[9] Giga, Y.;Miyakawa, T.; Osada, H. Two-dimensional Navier-Stokes flow with measures as initial vorticity. Arch. Rational Mech. Anal. 1988, 104, 223-250.

[10] Matsui, S.; Tokuno, S. Remark on fundamental solutions for vorticity equation of two dimensional Navier-Stokes flows, Hokkaido Math. J. 1997, 26, 529-539.

[11] Nash, J. Continuity of solutions of parabolic and elliptic equations. Amer. J. Math. 1958, 80, 931-954.

[12] Osada, H. Diffusion processes with generators of generalized divergence form. J. Math. Kyoto Univ. 1987, 27, 597-619. 